\title{
Decentralized Loan Management Application Using Smart Contracts on Block Chain
}

\author{
Arikumar K S ${ }^{\mathrm{a}, 1}$, Deepak Kumar A ${ }^{\mathrm{b}}$, GowthamC ${ }^{\mathrm{b}}$, SahayaBeniPrathiba ${ }^{\mathrm{c}}$ \\ ${ }^{\mathrm{a}, 1, \mathrm{~b}}$ St.Joseph's Institute of Technology, Chennai, India \\ ${ }^{\mathrm{c}}$ MIT Campus, Anna University, Chennai, India
}

\begin{abstract}
The most shocking events were was the recent discovery of the fraudulent activities in the Punjab National Bank. This is due to frequent systemic failures that detect human errors. Blockchain technology is the greatest solution for this issue. It is surprisingly common for the information settlement mechanism like SWIFT to be on a isolated ledger from the payment settlement mechanism. If the banks uses a ledger that stores information settlement distributed across all the participants, then the fraudulent user may reflect on all the available participants in the transactions, auditors and regulators. Our Paper is a Decentralised Loan Management Web Application (DApp) built on Ethereum blockchain which targets on preventing such fraudulent attacks on Loans sanctions by decentralising the processes. The security features authentication of the user identity, authentication of bank officials and multiple levels of verification of details are implemented using Public Key Infrastructure (PKI).
\end{abstract}

Keywords: BlockChain, Loan Management System, User Privacy, Smart Contracts.

\section{Introduction}

Banking security and privacy remains a major challenge. This brings in the new technology in the field of financial services industry in the form of blockchainbanking[1]. From greater user privacy and heightened security to lower processing fees and fewer errors, blockchain technology can make tremendous change in banking sector[2]. In this paper, the various core components and functions of the smart contract are outlined and analyzed. The proposed BC-based loan management is secure by thoroughly analyzing its security with respect to the fundamental security goals of confidentiality, integrity, and availability. The proposed system aims on preventing fraudulent attacks like PNB scam on Loans sanctions by decentralising the processes involved in Loan Sanctioning by using smart contracts and Ethereum Blockchain. Ethereum is an open-source, blockchain-based, platform which gives way for developing various Distributed Applications (DApps)for creating Smart Contracts. This platform provides advantages such as controlling frauds, system downtimes, interferences and the third-party frauds. Proof of authority and proof of work algorithms ensures that the data is real, authorized and immuted. 


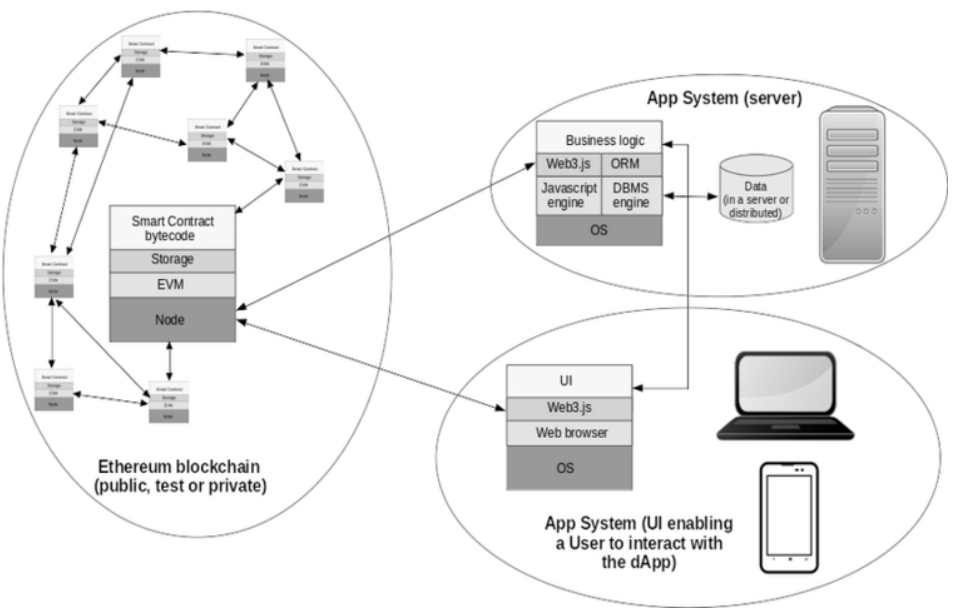

Figure.1. Architecture diagram for the proposed DApp system

\section{Related Works}

In this section, Hao Wang, Chaonian Guobet. al., designed a loan management system called LoC, which attempts to manage the loans based on smart contracts over permissioned blockchainHyperledger Fabric[3].Konstantinos Christidis et. al., designed Blockchains and Smart Contracts for the Internet of Things[4], which allows to have a distributed verifiable peer-to-peer network with non-trusting members can interact with each other without a trusted intermediary [5].Gareth W.Peterst et. al., designed an Internet of Money [6] and Hiroki Watanabe et. al., designed Block-chain Contract for securing a blockchain. This prevents the attacker for being controlling the resources, which in turn secures the blockchain [7].

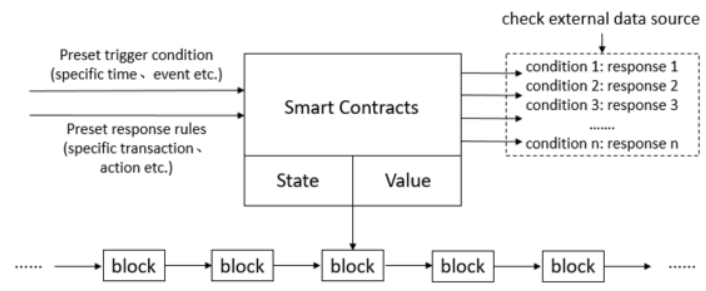

Figure.2. Mechanism of Smart Contract

\section{System Architecture}

Fig.1 depicts the flow of data and control among various operations of a Ethereum Decentralized Application. It also holds modules for authorization of nodes and verification and validation of resources concerned.Fig.2 depicts the working of the 
smart contracts for block creation and adding created blocks to the blockchain. Smart contracts help in exchanging valuables, shares, properties, and money without any conflicts. Furthermore, the system eliminates the intermediator called broker fares. The concept behind the smart contract can be easily described with the technology description of vending machine. The Smart Contracts in DApps enforces obligations in addition to defining rules and charging penalties for the created agreement.

\subsection{DApp System}

We have followed the approach of developing, implementing and testing the DApp in the ethereum simulator Ganache[8].Primarily, we launch the Ganache Ethereum Simulator using ganache-cli command in the command prompt. The simulator then creates 10 accounts which resemble the nodes in the blockchain. As the next step, connect to the Remix IDE by selecting the option Web3. Provider localhost:8545. Then start the Apache HTTP server and MySqlusingXAMPP and handle the administration using phpMyAdmin.

\subsection{Loan Requesting and Sanctioning Process}

The first UI of our DApp is the sign up / login page. Once the user logs in, the welcome page appears. Then he/she has to sign a message using their private key and this signature is then stored in the bank database. If login not available, the user creates an account in the Banking website by entering his/her Public Key along with the basic information for KYC. Then, the Loanee / Representative of an organisation has to digitally sign a fixed message(previously set by the bank and updated periodically) using his/her Private key before applying for loan or Loan.

If the verification is a success, then, the bank officials digitally sign the details of loan using their private key and upload the signed details in the blockchain. In case of loan, the block hash is stored in the user database. When the required number of bank officials authorize a particular loan(i.e) more block hashes are stored for a particular loan, the loan is sanctioned. Hence, multiple authentications and verifications are done before sanctioning loans thus augmenting more security by decentralizing the loan process[11-15].

\subsection{Loan Sanction Algorithm}

The Algorithm 1 is based on the loan sanction algorithm which is used at the backend of the loan management application to secure and ensure data integrity in the loan sanction process. The first parameter used in this algorithm is the user_type which has the type of the user. Sign method, verify method are used to verify the signature with the public key using digital signature algorithm. add_block creates a new block and stores the information passed to it in the block and adds the block to the block chain. Save method stores the parameter passed to it to the database. 
Algorithm 1

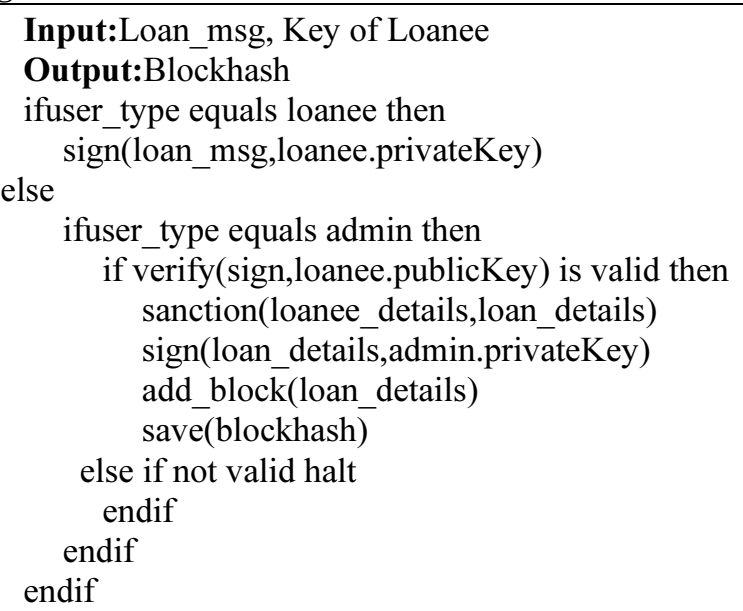

\section{Results and Discussions}

In this section, we present the simulation of our proposed system. The Backend code for the Banking Smart Contract is written in the language Solidity. The Solidity code written using the Solidity version 0.4.21[10].

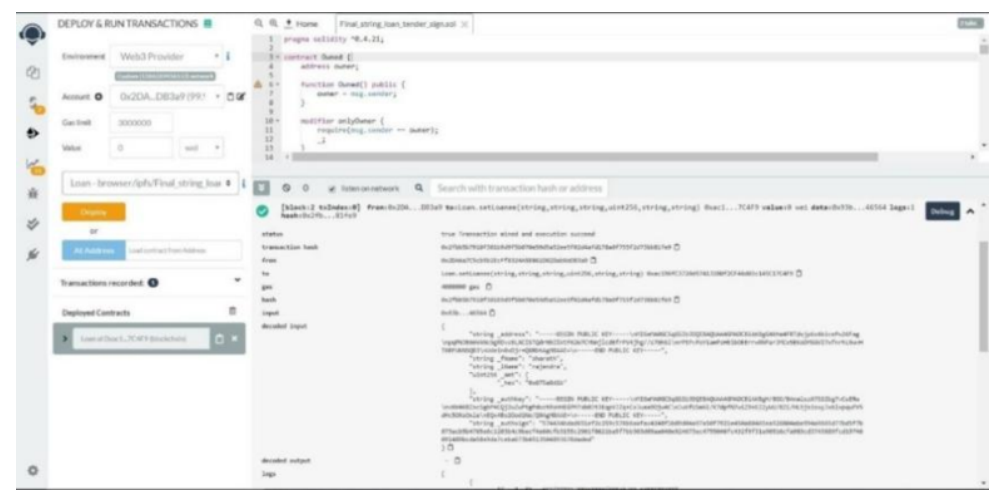

Figure.3.Storing Loan details in Blockchain and storing Blockhash in database

The Fig.3shows remix IDE, which shows the details of the added block of the blockchain. Remix is a browser-based compiler and IDE that enables users to build Ethereum contracts with Solidity language and to debug transactions. 


\section{Conclusion}

In this paper, we proposed a loan management system based on blockchain namely DApp. In real-time, the system securely shares the details about transactions by organizing the network, this action prevents the fraudsin the system. Our proposed system maintains the privacy of the valuable customers by eliminating the attackers or frauds who injecting vulnerable data. By our proposed system, the banks in India can be completely digitalized without any hesitation from the hackers and the attackers. Thus, the integration of blockchain in the loan management system incorporates easier, faster, and cheaper solutions, which can be adapted by the existing banking systems for experiencing the high-level security and privacy.

\section{References}

[1] Sachchidanand Singh and Nirmala Singh, Blockchain: Future of Financial and Cyber Security. $12,2017$.

[2] Adam Hahn and Rajveer Singh. Smart contract-based campus demonstration of decentralized transactive energy auctions.17(1), 2018.

[3] Hao Wang, ChaonianGuob and Shuhan Cheng. LoC - A new financial loan management system based on smart contracts

[4] Arikumar K.S., Natarajan V. (2021) FIoT: A QoS-Aware Fog-IoT Framework to Minimize Latency in IoT Applications via Fog Offloading. In: Bhateja V., Peng SL., Satapathy S.C., Zhang YD. (eds) Evolution in Computational Intelligence. Advances in Intelligent Systems and Computing, vol 1176.Springer, Singapore. https://doi.org/10.1007/978-981-15-5788-0_53

[5] Gareth W.Peterst and EfstathiosPanayi. Future of Transaction Processing and Smart Contracts on the Internet of Money. November 19, 2015.

[6] Hiroki Watanabe and Jay Kishigami. Blockchain Contract: Securing a block chain applied to Smart contracts. 20,2018.

[7] M. Ali, J. Nelson, R. Shea, and M. J. Freedman. (Feb. 2016).Block stack: Design and Implementation of a Global Naming SystemWith BC.

[8] Jiin- Chiou Cheng and Narn - Yih Lee. Blockchain and Smart Contract for Digital certificate. 21,2017.

[9] Maxim Ya.Afanasev and Yuri V. Fedosov.An application of block chain and smart contracts for machine-to-machine communication. May21,2017.

[10] Yi-HuiChenandShih-Hsinchen, Blockchainbasedsmartcontractforbiddingsystem, October 30,2016.

[11] S.Hema Kumar, J.UdayKiran, V.D.AKumar, G.Saranya, Ramalakshmi V.Effective Online Medical Appointment System. International Journal of Scientific \& Technology Research, Volume 8, Issue 09, September 2019, Pages $803-805$.

[12] V.D.Ambeth Kumar, Dr.M.Ramakrishnan, V.D.Ashok Kumar and Dr.S.Malathi (2015).Performance Improvement using an Automation System for Recognition of Multiple Parametric Features based on Human Footprint. for the International Journal of kuwait journal of science \& engineering, Vol 42, No 1 (2015), pp:109-132.

[13] V.D.AKumar, V.D.Ashok Kumar, S.Malathi, K.Vengatesan, M.Ramakrishnan.Facial Recognition System For Suspect Identification Using A Surveillance Camera. Pattern Recognition and Image Analysis (Springer), Volume 28, Issue 3, pp 410-420, 2018. (DOI: 10.1134/S1054661818030136)

[14] V.D.Ambeth Kumar and M.Ramakrishan.Employment Of Footprint Recognition System. in the month of December for Indian Journal of Computer Science and Engineering (IJCSE) Vol. 3 No.6 Dec 2013

[15]Ramya,T.,Dr.Malathi,S.,ratheeksha,G.R. and Dr.V.D.Ambeth Kumar (2014). Personalized authentication procedure for restricted web service access in mobile phones. Applications of Digital Information and Web Technologies (ICADIWT), 2014, Page(s):69 - 74, Bangalore, India (ISBN:978-1-4799-2258-1)

[16] B. Aravindh; V.D.Ambeth Kumar; G. Harish; V. Siddartth, " A novel graphical authentication system for secure banking systems", IEEE (ICSTM), Pages: $177-183$, 2-4 Aug. 2017, DOI: 10.1109/ICSTM.2017.8089147 